\title{
Linear Growth for Certain Elliptic Fibrations
}

\section{Pierre Le Boudec}

\section{EPFL SB MATHGEOM TAN, MA C3 604 (Bâtiment MA), Station 8, CH-1015 Lausanne, Switzerland}

Correspondence to be sent to: pierre.leboudec@epfl.ch

We prove that the number of rational points of bounded height on certain del Pezzo surfaces of degree 1 defined over $\mathbb{Q}$ grows linearly, as predicted by Manin's conjecture.

\section{Introduction}

\subsection{Rational points on elliptic fibrations}

The main goal of this article is to establish sharp bounds for the number of rational points of bounded height on certain del Pezzo surfaces of degree 1 defined over $\mathbb{Q}$. In their anticanonical embedding, these surfaces are defined by sextic forms in $\mathbb{P}(3,2,1,1)$. More precisely, they are isomorphic to a surface $V$ given by an equation of the shape

$$
y^{2}=x^{3}+F_{4}(u, v) x+F_{6}(u, v)
$$

where the coordinates in $\mathbb{P}(3,2,1,1)$ are denoted by $(y: x: u: v)$ to highlight the elliptic fibration and where $F_{4}, F_{6} \in \mathbb{Z}[u, v]$ are, respectively, a quartic and a sextic form such that $4 F_{4}^{3}+27 F_{6}^{2}$ is not identically 0 .

For $\mathbf{x}=(y: x: u: v) \in \mathbb{P}(3,2,1,1)(\mathbb{Q})$, we can choose coordinates $y, x, u, v \in \mathbb{Z}$ such that for every prime $p$, either $p \nmid u$ or $p \nmid v$ or $p^{2} \nmid x$ or $p^{3} \nmid y$. Then we can define an exponential height function $H: \mathbb{P}(3,2,1,1)(\mathbb{Q}) \rightarrow \mathbb{R}_{>0}$ by setting

$$
H(\mathbf{x})=\max \left\{|y|^{1 / 3},|x|^{1 / 2},|u|,|v|\right\} .
$$

Received July 30, 2013; Accepted November 20, 2014

(C) The Author(s) 2015. Published by Oxford University Press. All rights reserved. For permissions, please e-mail: journals.permissions@oup.com. 
For any Zariski open subset $U$ of $V$, we can introduce the number of rational points of bounded height on $U$, that is,

$$
N_{U, H}(B)=\#\{\mathbf{x} \in U(\mathbb{Q}), H(\mathbf{x}) \leq B\} .
$$

A conjecture of Manin (see [2]) predicts the asymptotic behavior of $N_{U, H}(B)$ as $B$ tends to $+\infty$, for some well-chosen Zariski open subset $U$ of $V$, but the current technology is very far from allowing us to approach it for any surface $V$. A weaker version states that $V$ has linear growth, by which we mean that there should exist an open subset $U$ of $V$ such that, for any fixed $\varepsilon>0$,

$$
N_{U, H}(B) \ll B^{1+\varepsilon} .
$$

The only authors who have addressed this problem seem to be Munshi $($ see $[7,8])$ and Mendes da Costa (see [6]).

More precisely, Mendes da Costa established that for any surface $V$ given by an equation of the shape (1.1), there exists $\delta>0$ such that $N_{V, H}(B) \ll B^{3-\delta}$, where the constant involved in the notation $\ll$ is independent of the forms $F_{4}$ and $F_{6}$. This bound is far from the expectation (1.2) but is not at all trivial, which illustrates the difficulty of this problem in general.

As already remarked by Munshi, it is easier to deal with certain specific examples of singular surfaces. The most striking result in Munshi's works is the following (see [8, Corollary 3]). Let $V_{\mathrm{e}, \lambda, R} \subset \mathbb{P}(3,2,1,1)$ be the surface defined by

$$
y^{2}=(x-e R(u, v))(x-\lambda R(u, v))(x-\bar{\lambda} R(u, v))
$$

where $e \in \mathbb{Z}, \lambda$ is a generator of the ring of integers of an imaginary quadratic field, and $R \in \mathbb{Z}[u, v]$ is a positive-definite quadratic form. Then we have

$$
N_{U_{\mathrm{e}, \lambda, R}, H}(B) \ll B^{5 / 4+\varepsilon},
$$

where $U_{\mathbf{e}, \lambda, R}$ is defined by removing from $V_{\mathbf{e}, \lambda, R}$ the subset defined by $y=0$. Although impressive, this result is still far from the conjectured upper bound (1.2).

Let $e_{1}, e_{2}, e_{3} \in \mathbb{Z}$ be three distinct integers and set $\mathbf{e}=\left(e_{1}, e_{2}, e_{3}\right)$. We also let $Q \in \mathbb{Z}[u, v]$ be a nondegenerate quadratic form. In this article, we are interested in the surfaces $V_{\mathrm{e}, Q} \subset \mathbb{P}(3,2,1,1)$ defined by

$$
y^{2}=\left(x-e_{1} Q(u, v)\right)\left(x-e_{2} Q(u, v)\right)\left(x-e_{3} Q(u, v)\right)
$$


We let $U_{\mathbf{e}, Q}$ be the open subset defined by removing from $V_{\mathbf{e}, Q}$ the two subsets given by $y=0$ and $Q(u, v)=0$. It is straightforward to check that all the surfaces defined by Equation (1.3) or (1.4) have two singularities of type $\mathbf{D}_{4}$ over $\overline{\mathbb{Q}}$.

Let us note that, all along this article, the constants involved in the notation « and $\gg$ may depend on $\varepsilon$, e, and $Q$.

The main result of this article is the following.

Theorem 1. Let $\varepsilon>0$ be fixed. We have the upper bound

$$
N_{U_{\mathrm{e}, Q}, H}(B) \ll B^{1+\varepsilon}
$$

As in the works of Munshi, the proof of Theorem 1 makes use of the natural elliptic fibration to parameterize the rational points on $U_{\mathrm{e}, Q}$. This leads us to investigate integral points of bounded height on quadratic twists of a fixed elliptic curve with full rational 2-torsion. This is the purpose of Section 1.2.

It is worth mentioning that the analysis of the parameterization of the rational points given by Munshi in [8] shows that it should be easy to adapt Lemma 3 (see Section 2.2) to prove that the surfaces defined by (1.3), and considered by Munshi, also have linear growth.

Another interesting problem is to prove sharp lower bounds for $N_{U_{\mathrm{e}, Q}, H}(B)$. A simpler way to state this is to ask what can be said about the quantity

$$
\beta_{U_{\mathrm{e}, Q}}(B)=\frac{\log N_{U_{\mathrm{e}, Q}, H}(B)}{\log B} .
$$

In the following, we choose to take $Q(u, v)=u v$, even though similar results could be proved for other choices of $Q$. We, respectively, call $V_{\mathrm{e}}$ and $U_{\mathrm{e}}$ the surface and the open subset corresponding to this choice. We establish the following result.

Corollary 1. The limit of $\beta_{U_{\mathrm{e}}}(B)$ as $B$ tends to $+\infty$ exists and equals 1 . More precisely, we have

$$
\beta_{U_{\mathrm{e}}}(B)=1+O\left(\frac{1}{\log \log B}\right) .
$$

To prove the lower bound $B(\log B)^{8} \ll N_{U_{\mathrm{e}}, H}(B)$, which is conjecturally best possible, a natural idea is to make use of universal torsors above $V_{\mathrm{e}}$. Indeed, this strategy has been successful to establish Manin's conjecture for several examples of singular del Pezzo surfaces of low degree (see [1,4] for the most striking results). Hausen and Süss [3, Example 5.5] have computed the equations of such a torsor and it turns out that proving this lower bound does not seem to be easy. It would be interesting to solve this problem. 


\subsection{Integral points on quadratic twists}

For $n \geq 1$, we introduce the elliptic curve $E_{n, \mathbf{e}}$ defined by the equation

$$
y^{2}=\left(x-e_{1} n\right)\left(x-e_{2} n\right)\left(x-e_{3} n\right) .
$$

We instantly check that the curves $E_{1, \mathrm{e}}$ and $E_{n, \mathrm{e}}$ are isomorphic over $\mathbb{Q}(\sqrt{n})$.

Let $P_{\infty}$ be the point at infinity on $E_{n, \mathbf{e}}$. Given a point $P \in E_{n, \mathbf{e}}(\mathbb{Q}) \backslash\left\{P_{\infty}\right\}$, we denote its coordinates by $(x, y)$. Our interest lies in the set of integral points on $E_{n, \mathbf{e}}$, so we define

$$
E_{n, \mathbf{e}}(\mathbb{Z})=\left\{P \in E_{n, \mathbf{e}}(\mathbb{Q}) \backslash\left\{P_{\infty}\right\}, x \in \mathbb{Z}\right\},
$$

and also

$$
E_{n, \mathbf{e}}^{*}(\mathbb{Z})=\left\{P \in E_{n, \mathbf{e}}(\mathbb{Z}), y \neq 0\right\} .
$$

The elements of $E_{n, \mathbf{e}}^{*}(\mathbb{Z})$ will be referred to as the nontrivial integral points on $E_{n, \mathbf{e}}$.

A difficult problem is to obtain upper bounds for the cardinality of the set of $n \leq N$ such that $E_{n, \mathbf{e}}$ has at least one nontrivial integral point. It is reasonable to expect that this set has density 0 but the proof of this statement seems to be out of reach. An easier problem is to investigate this question for integral points of bounded height.

Given $P \in E_{n, \mathbf{e}}(\mathbb{Z})$, we define its exponential naive height $\mathcal{H}(P)$ by setting

$$
\mathcal{H}(P)=\max \left\{|y|^{1 / 3},|x|^{1 / 2}\right\} .
$$

The following result will be the key tool in the proof of Theorem 1. It gives lower and upper bounds for the number of nontrivial integral points of bounded height on the curves $E_{n, \mathrm{e}}$ on average over $n$.

Proposition 1. We have the bounds

$$
B \ll \sum_{n \geq 1} \#\left\{P \in E_{n, \mathbf{e}}^{*}(\mathbb{Z}), \mathcal{H}(P) \leq B\right\} \ll B(\log B)^{\delta_{\mathbf{e}}},
$$

where $\delta_{\mathbf{e}}=4$ if $e_{1} e_{2} e_{3} \neq 0$ and $\delta_{\mathbf{e}}=6$ otherwise.

Note that the interest of Proposition 1 mainly lies in the upper bound, and the lower bound implies that it is sharp up to the factor $(\log B)^{\delta_{e}}$.

One can immediately check that there exist integers $n \gg B^{2}$ for which the set $\left\{P \in E_{n, \mathbf{e}}^{*}(\mathbb{Z}), \mathcal{H}(P) \leq B\right\}$ is not empty. Therefore, the upper bound in Proposition 1 states that most quadratic twists of $E_{1, \mathrm{e}}$ do not have a nontrivial integral point of bounded height. 


\subsection{Outline of the article}

We start by establishing Proposition 1. The proof of this result goes in two steps. The first step consists in using the fact that $E_{n, \mathbf{e}}$ has full rational 2-torsion to parameterize the integral points on $E_{n, \mathbf{e}}$ using a complete 2-descent. This is achieved in Section 2.1. In the second step, we bound the number of nontrivial integral points of bounded height on the curves $E_{n, \mathrm{e}}$ on average over $n$. To achieve this, we appeal to the recent result of the author [5, Lemma 4]. This lemma is stated in Section 2.2.

Then, we prove Theorem 1 using the natural elliptic fibration and the upper bound in Proposition 1. Finally, Corollary 1 also follows from this upper bound, together with the lower bound $B \ll N_{U_{\mathrm{e}}, H}(B)$.

\section{Preliminaries}

\subsection{Descent argument}

In this section, we derive a convenient parameterization of the integral points on $E_{n, \mathbf{e}}$ using the fact that $E_{n, \mathrm{e}}$ has full rational 2-torsion. We start by proving the following elementary lemma.

Lemma 1. Let $\left(y, x_{1}, x_{2}, x_{3}\right) \in \mathbb{Z}_{\neq 0}^{4}$ be such that $y^{2}=x_{1} x_{2} x_{3}$. There exists a unique way to write

$$
x_{i}=d_{j} d_{k} w^{2} a_{i}^{2} a_{j} a_{k} b_{i}^{2}
$$

for $\{i, j, k\}=\{1,2,3\}$ and

$$
y=d_{1} d_{2} d_{3} w^{3} a_{1}^{2} a_{2}^{2} a_{3}^{2} b_{1} b_{2} b_{3}
$$

where $\left(d_{1}, d_{2}, d_{3}, w, a_{1}, a_{2}, a_{3}, b_{1}, b_{2}, b_{3}\right) \in \mathbb{Z}_{\neq 0}^{4} \times \mathbb{Z}_{>0}^{6}$ is subject to the conditions $\left|\mu\left(a_{i}\right)\right|=1$ and $\operatorname{gcd}\left(d_{i} a_{j} b_{j}, d_{j} a_{i} b_{i}\right)=1$ for $i, j \in\{1,2,3\}, i \neq j$, and $d_{1} d_{2} d_{3}>0$.

Proof. Let us set $x=\operatorname{gcd}\left(x_{1}, x_{2}, x_{3}\right)$ and let us write $x_{i}=x x_{i}^{\prime}$ for $i \in\{1,2,3\}$, where $\operatorname{gcd}\left(x_{1}^{\prime}, x_{2}^{\prime}, x_{3}^{\prime}\right)=1$. We see that $x \mid y$ and we can thus write $y=x y^{\prime}$. We obtain

$$
y^{2}=x x_{1}^{\prime} x_{2}^{\prime} x_{3}^{\prime}
$$

Let us now set $d_{i}=\operatorname{sign}\left(x_{i}^{\prime}\right) \operatorname{gcd}\left(x_{j}^{\prime}, x_{k}^{\prime}\right)$ for $\{i, j, k\}=\{1,2,3\}$. Let us note that we have $d_{1} d_{2} d_{3}>0$. We can write $x_{i}^{\prime}=d_{j} d_{k} \xi_{i}$ with $\xi_{i}>0$ for $\{i, j, k\}=\{1,2,3\}$, where $\operatorname{gcd}\left(d_{i} \xi_{j}, d_{j} \xi_{i}\right)=1$ for $i, j \in\{1,2,3\}, i \neq j$. Since $d_{1} d_{2} d_{3} \mid y^{\prime}$, we can write $y^{\prime}=d_{1} d_{2} d_{3} z$. We 
thus get

$$
z^{2}=x \xi_{1} \xi_{2} \xi_{3}
$$

There is a unique way to write $\xi_{i}=a_{i} b_{i}^{2}$ with $a_{i}, b_{i}>0$ and $\left|\mu\left(a_{i}\right)\right|=1$ for $i \in\{1,2,3\}$. We see that $b_{1} b_{2} b_{3} \mid z$ so we can write $z=b_{1} b_{2} b_{3} z$. We finally obtain

$$
z^{2}=x a_{1} a_{2} a_{3}
$$

Since $a_{1}, a_{2}$, and $a_{3}$ are squarefree and pairwise coprime, we can write $x=w^{2} a_{1} a_{2} a_{3}$ and $z^{\prime}=w a_{1} a_{2} a_{3}$, which completes the proof.

Lemma 1 immediately implies the following result, which provides us with the desired parameterization of the nontrivial integral points on $E_{n, \mathbf{e}}$.

Lemma 2. There is a bijection between the set of nontrivial integral points on $E_{n, \mathrm{e}}$ and the set of $\left(d_{1}, d_{2}, d_{3}, w, a_{1}, a_{2}, a_{3}, b_{1}, b_{2}, b_{3}\right) \in \mathbb{Z}_{\neq 0}^{4} \times \mathbb{Z}_{>0}^{6}$ satisfying, for $\{i, j, k\}=\{1,2,3\}$, the equations

$$
\left(e_{i}-e_{j}\right) n=d_{k} w^{2} a_{1} a_{2} a_{3}\left(d_{i} a_{j} b_{j}^{2}-d_{j} a_{i} b_{i}^{2}\right),
$$

and the conditions $\left|\mu\left(a_{i}\right)\right|=1$ and $\operatorname{gcd}\left(d_{i} a_{j} b_{j}, d_{j} a_{i} b_{i}\right)=1$ for $i, j \in\{1,2,3\}, i \neq j$, and $d_{1} d_{2} d_{3}>0$. This bijection is given, for $P \in E_{n, \mathbf{e}}^{*}(\mathbb{Z})$ with coordinates $(x, y) \in \mathbb{Z}^{2}$, by

$$
\begin{aligned}
& x=e_{i} n+d_{j} d_{k} w^{2} a_{i}^{2} a_{j} a_{k} b_{i}^{2}, \\
& y=d_{1} d_{2} d_{3} w^{3} a_{1}^{2} a_{2}^{2} a_{3}^{2} b_{1} b_{2} b_{3},
\end{aligned}
$$

for $\{i, j, k\}=\{1,2,3\}$.

\subsection{Geometry of numbers}

The following lemma follows from the recent work of the author [5, Lemma 4]. It draws upon both geometry of numbers and analytic number theory tools, and will be the key result in the proof of Proposition 1.

Lemma 3. Let $\mathbf{f}=\left(f_{1}, f_{2}, f_{3}\right) \in \mathbb{Z}_{\neq 0}^{3}$ be a vector satisfying the conditions $\operatorname{gcd}\left(f_{i}, f_{j}\right)=1$ for $i, j \in\{1,2,3\}, i \neq j$, and let $U_{i}, V_{i} \geq 1$ for $i \in\{1,2,3\}$. Let also $N_{\mathrm{f}}=N_{\mathrm{f}}\left(U_{1}, U_{2}, U_{3}, V_{1}, V_{2}, V_{3}\right)$ be the number of vectors $\left(u_{1}, u_{2}, u_{3}\right) \in \mathbb{Z}_{\neq 0}^{3}$ and $\left(v_{1}, v_{2}, v_{3}\right) \in \mathbb{Z}_{\neq 0}^{3}$ satisfying $\left|u_{i}\right| \leq U_{i},\left|v_{i}\right| \leq V_{i}$ 
for $i \in\{1,2,3\}$, and the equation

$$
f_{1} u_{1} v_{1}^{2}+f_{2} u_{2} v_{2}^{2}+f_{3} u_{3} v_{3}^{2}=0
$$

and such that $\operatorname{gcd}\left(u_{i} v_{i}, u_{j} v_{j}\right)=1$ for $i, j \in\{1,2,3\}, i \neq j$. Let $\varepsilon>0$ be fixed. We have the bound

$$
N_{\mathrm{f}} \ll_{\mathrm{f}}\left(U_{1} U_{2} U_{3}\right)^{2 / 3}\left(V_{1} V_{2} V_{3}\right)^{1 / 3} M_{\varepsilon}\left(U_{1}, U_{2}, U_{3}\right),
$$

where

$$
M_{\varepsilon}\left(U_{1}, U_{2}, U_{3}\right)=1+\max _{\{i, j, k\}=\{1,2,3\}}\left(U_{i} U_{j}\right)^{-1 / 2+\varepsilon} \log 2 U_{k}
$$

\section{Integral Points on Quadratic Twists}

\subsection{Proof of Proposition 1}

Let us start by proving the upper bound in Proposition 1. Lemma 2 asserts that $(y, x) \in$ $\mathbb{Z}_{\neq 0} \times \mathbb{Z}$ satisfies the equation

$$
y^{2}=\left(x-e_{1} n\right)\left(x-e_{2} n\right)\left(x-e_{3} n\right)
$$

if and only if $x$ and $y$ can be written, for $\{i, j, k\}=\{1,2,3\}$, as

$$
\begin{aligned}
& x=e_{i} n+d_{j} d_{k} w^{2} a_{i}^{2} a_{j} a_{k} b_{i}^{2}, \\
& y=d_{1} d_{2} d_{3} w^{3} a_{1}^{2} a_{2}^{2} a_{3}^{2} b_{1} b_{2} b_{3},
\end{aligned}
$$

where $\left(d_{1}, d_{2}, d_{3}, w, a_{1}, a_{2}, a_{3}, b_{1}, b_{2}, b_{3}\right) \in \mathbb{Z}_{\neq 0}^{4} \times \mathbb{Z}_{>0}^{6}$ satisfies, for $\{i, j, k\}=\{1,2,3\}$, the equations

$$
\left(e_{i}-e_{j}\right) n=d_{k} w^{2} a_{1} a_{2} a_{3}\left(d_{i} a_{j} b_{j}^{2}-d_{j} a_{i} b_{i}^{2}\right)
$$

and the conditions $\left|\mu\left(a_{i}\right)\right|=1$ and $\operatorname{gcd}\left(d_{i} a_{j} b_{j}, d_{j} a_{i} b_{i}\right)=1$ for $i, j \in\{1,2,3\}, i \neq j$, and $d_{1} d_{2} d_{3}>0$. Equations (3.2) can have a solution $n \in \mathbb{Z}_{>0}$ only if

$$
\left(e_{2}-e_{3}\right) d_{2} d_{3} a_{1} b_{1}^{2}+\left(e_{3}-e_{1}\right) d_{1} d_{3} a_{2} b_{2}^{2}+\left(e_{1}-e_{2}\right) d_{1} d_{2} a_{3} b_{3}^{2}=0
$$

Moreover, since $e_{1}, e_{2}$, and $e_{3}$ are distinct, there is at most one such solution $n \in$ $\mathbb{Z}_{>0}$. Let us call $h=\operatorname{gcd}\left(e_{2}-e_{3}, e_{3}-e_{1}, e_{1}-e_{2}\right)$. The conditions $\operatorname{gcd}\left(d_{i}, d_{j} a_{i} b_{i}\right)=1$ and 
Equation (3.3) imply that $d_{i} \mid\left(e_{j}-e_{k}\right) / h$ for $\{i, j, k\}=\{1,2,3\}$ so we can write $e_{2}-e_{3}=$ $h d_{1} f_{1}, e_{3}-e_{1}=h d_{2} f_{2}$, and $e_{1}-e_{2}=h d_{3} f_{3}$, and we have $\operatorname{gcd}\left(f_{1}, f_{2}, f_{3}\right)=1$. From the two relations $d_{1} f_{1}+d_{2} f_{2}+d_{3} f_{3}=0$ and

$$
f_{1} a_{1} b_{1}^{2}+f_{2} a_{2} b_{2}^{2}+f_{3} a_{3} b_{3}^{2}=0
$$

and the conditions $\operatorname{gcd}\left(d_{i}, a_{i} b_{i}\right)=1$ for $i \in\{1,2,3\}$, we deduce that $\operatorname{gcd}\left(f_{i}, f_{j}\right)=1$ for $i, j \in$ $\{1,2,3\}, i \neq j$.

From now on, we use the notation $\mathbf{f}=\left(f_{1}, f_{2}, f_{3}\right)$. We let $\mathcal{N}_{\mathbf{f}}(B)$ be the number of $\left(w, a_{1}, a_{2}, a_{3}, b_{1}, b_{2}, b_{3}\right) \in \mathbb{Z}_{\neq 0} \times \mathbb{Z}_{>0}^{6}$ satisfying Equation (3.4), the inequality

$$
|w|^{3} a_{1}^{2} a_{2}^{2} a_{3}^{2} b_{1} b_{2} b_{3} \leq B^{3}
$$

and the conditions $\operatorname{gcd}\left(a_{i} b_{i}, a_{j} b_{j}\right)=1$ for $i, j \in\{1,2,3\}, i \neq j$. The investigation above shows that

$$
\sum_{n \geq 1} \#\left\{P \in E_{n, \mathbf{e}}^{*}(\mathbb{Z}), \mathcal{H}(P) \leq B\right\} \ll \max _{\mathbf{f}} \mathcal{N}_{\mathbf{f}}(B)
$$

where the maximum is taken over f satisfying $f_{i} \mid\left(e_{j}-e_{k}\right) / h$ for $\{i, j, k\}=\{1,2,3\}$ and $\operatorname{gcd}\left(f_{i}, f_{j}\right)=1$ for $i, j \in\{1,2,3\}, i \neq j$.

We have thus proved that it is sufficient for our purpose to bound the quantity $\mathcal{N}_{\mathbf{f}}(B)$. To achieve this, for $i \in\{1,2,3\}$, we let $W, A_{i}, B_{i} \geq \frac{1}{2}$ run over the set of powers of 2 and we define $\mathcal{M}_{\mathbf{f}}=\mathcal{M}_{\mathbf{f}}\left(W, A_{1}, A_{2}, A_{3}, B_{1}, B_{2}, B_{3}\right)$ as the number of $\left(w, a_{1}, a_{2}, a_{3}, b_{1}, b_{2}, b_{3}\right) \in \mathbb{Z}_{\neq 0} \times \mathbb{Z}_{>0}^{6}$ satisfying Equation (3.4), the conditions $W<|w| \leq 2 W$, $A_{i}<a_{i} \leq 2 A_{i}$, and $B_{i}<b_{i} \leq 2 B_{i}$, and $\operatorname{gcd}\left(a_{i} b_{i}, a_{j} b_{j}\right)=1$ for $i, j \in\{1,2,3\}, i \neq j$. We have

$$
\mathcal{N}_{\mathbf{f}}(B) \ll \sum_{\substack{W, A_{i}, B_{i} \\ i \in\{1,2,3\}}} \mathcal{M}_{\mathbf{f}}
$$

where the sum is over $W, A_{i}, B_{i} \geq \frac{1}{2}, i \in\{1,2,3\}$, satisfying the inequality

$$
W^{3} A_{1}^{2} A_{2}^{2} A_{3}^{2} B_{1} B_{2} B_{3} \leq B^{3}
$$

Lemma 3 gives the upper bound

$$
\mathcal{M}_{\mathbf{f}} \ll W\left(A_{1} A_{2} A_{3}\right)^{2 / 3}\left(B_{1} B_{2} B_{3}\right)^{1 / 3} M_{\varepsilon}\left(A_{1}, A_{2}, A_{3}\right),
$$


where $M_{\varepsilon}\left(A_{1}, A_{2}, A_{3}\right)$ is defined in Lemma 3. Choosing for instance $\varepsilon=1 / 4$ and summing over $W$ using the condition (3.5), we finally obtain

$$
\begin{aligned}
\mathcal{N}_{\mathbf{f}}(B) & \ll \sum_{\substack{W, A_{i}, B_{i} \\
i \in\{1,2,3\}}} W\left(A_{1} A_{2} A_{3}\right)^{2 / 3}\left(B_{1} B_{2} B_{3}\right)^{1 / 3} M_{1 / 4}\left(A_{1}, A_{2}, A_{3}\right) \\
& \ll B \sum_{\substack{A_{i}, B_{i} \\
i \in\{1,2,3\}}} M_{1 / 4}\left(A_{1}, A_{2}, A_{3}\right) \\
& \ll B(\log B)^{6},
\end{aligned}
$$

which completes the first part of the proof of the upper bound in Proposition 1.

Now let us assume that $e_{1} e_{2} e_{3} \neq 0$ and let us prove that we can take $\delta_{\mathrm{e}}=4$ in Proposition 1. If $n>2 B^{2}$, then, since

$$
x=e_{i} n+d_{j} d_{k} w^{2} a_{i}^{2} a_{j} a_{k} b_{i}^{2}
$$

for $\{i, j, k\}=\{1,2,3\},|x| \leq B^{2}$, and $e_{1} e_{2} e_{3} \neq 0$, we have $\left|d_{j} d_{k}\right| w^{2} a_{i}^{2} a_{j} a_{k} b_{i}^{2}>B^{2}$ for $\{i, j, k\}=$ $\{1,2,3\}$, but this is in contradiction with $|y| \leq B^{3}$. This implies that $\left\{P \in E_{n, \mathbf{e}}^{*}(\mathbb{Z}), \mathcal{H}(P) \leq\right.$ $B\}$ is empty provided that $n>2 B^{2}$ so we can assume that $n \leq 2 B^{2}$. Therefore, for $\{i, j, k\}=$ $\{1,2,3\}$, we get the conditions

$$
w^{2} a_{i}^{2} a_{j} a_{k} b_{i}^{2} \ll B^{2}
$$

We now proceed similarly as in the first case. We let $\mathcal{N}_{\mathbf{f}}^{\prime}(B)$ be the number of $\left(w, a_{1}, a_{2}, a_{3}, b_{1}, b_{2}, b_{3}\right) \in \mathbb{Z}_{\neq 0} \times \mathbb{Z}_{>0}^{6}$ satisfying Equation (3.4), the inequalities (3.6), and the conditions $\operatorname{gcd}\left(a_{i} b_{i}, a_{j} b_{j}\right)=1$ for $i, j \in\{1,2,3\}, i \neq j$. Once again, it is sufficient for our purpose to bound $\mathcal{N}_{\mathbf{f}}^{\prime}(B)$, and we have

$$
\mathcal{N}_{\mathbf{f}}^{\prime}(B) \ll \sum_{\substack{W, A_{i}, B_{i} \\ i \in\{1,2,3\}}} W\left(A_{1} A_{2} A_{3}\right)^{2 / 3}\left(B_{1} B_{2} B_{3}\right)^{1 / 3} M_{1 / 4}\left(A_{1}, A_{2}, A_{3}\right)
$$

where the sum is over $W, A_{i}, B_{i} \geq \frac{1}{2}, i \in\{1,2,3\}$, running over the set of powers of 2 and satisfying, for $\{i, j, k\}=\{1,2,3\}$, the inequalities

$$
W^{2} A_{i}^{2} A_{j} A_{k} B_{i}^{2} \ll B^{2} .
$$


Summing over $B_{i}, i \in\{1,2,3\}$, using the conditions (3.7), we get

$$
\begin{aligned}
\mathcal{N}_{\mathbf{f}}^{\prime}(B) & \ll B \sum_{\substack{W, A_{i} \\
i \in\{1,2,3\}}} M_{1 / 4}\left(A_{1}, A_{2}, A_{3}\right) \\
& \ll B(\log B)^{4},
\end{aligned}
$$

as claimed.

Let us now prove the lower bound in Proposition 1. We can assume by symmetry that $e_{3}>\max \left\{e_{1}, e_{2}\right\}$ and thus $2 e_{3}-e_{1}-e_{2}>0$. We note that if $n$ can be written as $n=$ $2\left(2 e_{3}-e_{1}-e_{2}\right) w^{2}$ for some $w \in \mathbb{Z}_{>0}$, then the equalities

$$
\begin{aligned}
& x=2\left(-2 e_{1} e_{2}+e_{1} e_{3}+e_{2} e_{3}\right) w^{2}, \\
& y=4\left(e_{1}-e_{2}\right)\left(e_{2}-e_{3}\right)\left(e_{3}-e_{1}\right) w^{3},
\end{aligned}
$$

define a point $P \in E_{n, \mathbf{e}}^{*}(\mathbb{Z})$. Moreover, this point satisfies $\mathcal{H}(P) \leq B$ provided that $w \ll B$. Therefore, we have

$$
\sum_{n \geq 1} \#\left\{P \in E_{n, \mathbf{e}}^{*}(\mathbb{Z}), \mathcal{H}(P) \leq B\right\} \gg B
$$

which completes the proof of Proposition 1.

\section{Rational Points on Elliptic Fibrations}

\subsection{Proof of Theorem 1}

Recall that $V_{\mathrm{e}, Q} \subset \mathbb{P}(3,2,1,1)$ is defined by the equation

$$
y^{2}=\left(x-e_{1} Q(u, v)\right)\left(x-e_{2} Q(u, v)\right)\left(x-e_{3} Q(u, v)\right) .
$$

Thus, we have

$$
\begin{aligned}
N_{U_{\mathrm{e}, Q}, H}(B) & \ll \sum_{\substack{|u|,|v| \leq B \\
Q(u, v) \neq 0}} \#\left\{(y, x) \in \mathbb{Z}_{\neq 0} \times \mathbb{Z},|y| \leq B^{3},|x| \leq B^{2},(4.1)\right\} \\
& \ll \sum_{n \in \mathbb{Z}_{\neq 0}} \#\left\{(y, x) \in \mathbb{Z}_{\neq 0} \times \mathbb{Z},|y| \leq B^{3},|x| \leq B^{2},(3.1)\right\} \sum_{\substack{|u|,|v| \leq B \\
Q(u, v)=n}} 1 .
\end{aligned}
$$

Since $Q$ is nondegenerate, we have

$$
\#\left\{(u, v) \in \mathbb{Z}^{2},|u|,|v| \leq B, Q(u, v)=n\right\} \ll B^{\varepsilon} .
$$


As a result, we get

$$
N_{U_{\mathrm{e}, Q}, H}(B) \ll B^{\varepsilon} \sum_{n \in \mathbb{Z}_{\neq 0}} \#\left\{P \in E_{n, \mathbf{e}}^{*}(\mathbb{Z}), \mathcal{H}(P) \leq B\right\} .
$$

We note that the sum in the right-hand side can be rewritten as

$$
\sum_{n \geq 1} \#\left\{P \in E_{n, \mathbf{e}}^{*}(\mathbb{Z}), \mathcal{H}(P) \leq B\right\}+\sum_{n \geq 1} \#\left\{P \in E_{n,-\mathbf{e}}^{*}(\mathbb{Z}), \mathcal{H}(P) \leq B\right\} .
$$

Therefore, using twice the upper bound in Proposition 1, we obtain

$$
N_{U_{\mathrm{e}, Q}, H}(B) \ll B^{1+\varepsilon},
$$

which ends the proof of Theorem 1 .

\subsection{Proof of Corollary 1}

We proceed exactly as in the proof of Theorem 1 . We have

$$
N_{U_{\mathrm{e}}, H}(B) \ll \sum_{n \in \mathbb{Z}_{\neq 0}} \#\left\{(y, x) \in \mathbb{Z}_{\neq 0} \times \mathbb{Z},|y| \leq B^{3},|x| \leq B^{2},(3.1)\right\} \sum_{\substack{|u|,|v| \leq B \\ w v=n}} 1 .
$$

Then, if $n \leq B^{2}$, we have

$$
\begin{aligned}
\#\left\{(u, v) \in \mathbb{Z}^{2},|u|,|v| \leq B, u v=n\right\} & \leq 2 \tau(n) \\
& \ll n^{1 / \log \log n} \\
& \ll B^{2 / \log \log B},
\end{aligned}
$$

and this upper bound also holds if $n>B^{2}$. This shows that

$$
N_{U_{\mathrm{e}}, H}(B) \ll B^{2 / \log \log B} \sum_{n \in \mathbb{Z}_{\neq 0}} \#\left\{P \in E_{n, \mathbf{e}}^{*}(\mathbb{Z}), \mathcal{H}(P) \leq B\right\} .
$$

As in the proof of Theorem 1, using twice the upper bound in Proposition 1, we obtain

$$
N_{U_{\mathrm{e}}, H}(B) \ll B^{1+3 / \log \log B}
$$

Let us now prove a lower bound for $N_{U_{\mathrm{e}}, H}(B)$. Let us assume by symmetry that $e_{3}>\max \left\{e_{1}, e_{2}\right\}$ so that $2 e_{3}-e_{1}-e_{2}>0$, and let us denote by $v_{2}(m)$ the 2-adic valuation of an integer $m \geq 1$. Let $\mathcal{R}_{\mathbf{e}}(B)$ be the number of $(y, x, u, v) \in \mathbb{Z}_{\neq 0}^{4}$ such that 
$\max \left\{|y|^{1 / 3},|x|^{1 / 2},|u|,|v|\right\} \leq B$ and which can be written as

$$
\begin{aligned}
& y=4\left(e_{1}-e_{2}\right)\left(e_{2}-e_{3}\right)\left(e_{3}-e_{1}\right) w_{1}^{3} w_{2}^{3}, \\
& x=2\left(-2 e_{1} e_{2}+e_{1} e_{3}+e_{2} e_{3}\right) w_{1}^{2} w_{2}^{2}, \\
& u=2^{1+v_{2}\left(2 e_{3}-e_{1}-e_{2}\right)} w_{1}^{2}, \\
& v=\left(2 e_{3}-e_{1}-e_{2}\right) 2^{-v_{2}\left(2 e_{3}-e_{1}-e_{2}\right)} w_{2}^{2},
\end{aligned}
$$

where $\left(w_{1}, w_{2}\right) \in \mathbb{Z}_{>0}^{2}$ satisfies $\operatorname{gcd}\left(w_{1},\left(2 e_{3}-e_{1}-e_{2}\right) w_{2}\right)=\operatorname{gcd}\left(w_{2}, 2\right)=1$. Since $\operatorname{gcd}(u, v)=$ 1 in the parameterization above, it is immediate to check that

$$
N_{U_{\mathrm{e}}, H}(B) \geq \mathcal{R}_{\mathbf{e}}(B) .
$$

Since we clearly have $\mathcal{R}_{\mathrm{e}}(B) \gg B$, we have obtained the lower bound

$$
B \ll N_{U_{\mathrm{e}}, H}(B) .
$$

Let us note that improving this lower bound by a few $\log B$ factors would not be hard. However, as already explained in Section 1, proving the lower bound of the expected order of magnitude for $N_{U_{\mathrm{e}}, H}(B)$ does not seem to be easy.

Recalling the definition (1.5) of $\beta_{U_{\mathrm{e}}}(B)$, we see that the two bounds (4.2) and (4.3) complete the proof of Corollary 1 .

\section{Acknowledgements}

It is a pleasure for the author to thank Régis de la Bretèche, Timothy Browning, Daniel Loughran, Dave Mendes da Costa, Peter Sarnak, Arul Shankar, and Anders Södergren for interesting and stimulating conversations related to the topics of this article.

\section{Funding}

The financial support and the perfect working conditions provided by the Institute for Advanced Study are gratefully acknowledged. This material is based upon work supported by the National Science Foundation under agreement No. DMS-1128155. Any opinions, findings and conclusions or recommendations expressed in this material are those of the author and do not necessarily reflect the views of the National Science Foundation.

\section{References}

[1] Baier, S. and T. D. Browning. "Inhomogeneous cubic congruences and rational points on del Pezzo surfaces." Journal für die Reine und Angewandte Mathematik 680 (2013): 69-151. 
[2] Franke, J., Y. I. Manin, and Y. Tschinkel. "Rational points of bounded height on Fano varieties." Inventiones Mathematicae 95, no. 2 (1989): 421-35.

[3] Hausen, J. and H. Süß. "The Cox ring of an algebraic variety with torus action." Advances in Mathematics 225, no. 2 (2010): 977-1012.

[4] Le Boudec, P. "Affine congruences and rational points on a certain cubic surface." Algebra $\mathcal{S}$ Number Theory 8, no. 5 (2014): 1259-96.

[5] Le Boudec, P. "Density of rational points on a certain smooth bihomogeneous threefold." International Mathematics Research Notices 2015, no. 21 (2015): 10703-15.

[6] Mendes da Costa, D. "Integral points on elliptic curves and the Bombieri-Pila bounds." (2013): preprint arXiv:1301.4116v2.

[7] Munshi, R. "Density of rational points on elliptic fibrations." Acta Arithmetica 129, no. 1 (2007): 63-70.

[8] Munshi, R. "Density of rational points on elliptic fibrations. II." Acta Arithmetica 134, no. 2 (2008): 133-40. 